\title{
L. The method of least squares
}

\section{F.Y. Edgeworth H.A.}

To cite this article: F.Y. Edgeworth H.A. (1883) L. The method of least squares, Philosophical Magazine Series 5, 16:101, 360-375, DOI: 10.1080/14786448308627445

To link to this article: http://dx.doi.org/10.1080/14786448308627445

曲 Published online: 28 Apr 2009.

Submit your article to this journal 정

LII Article views: 11

Q View related articles $₫$

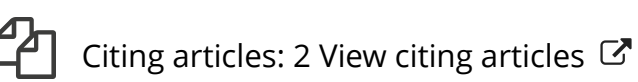


The opinion that the great mass of the ice on the Antarctic continent and also on Greenland lies near to the outer edge, and that it gradually diminishes inwards till at last it disappears, is evidently one based on a misapprehension as to the physical conditions of continental ice. I cannot help believing that had Professor Nordenskjöld duly reflected on the necessary physical and mechanical conditions of the problem which he is endeavouring to solve, he would not have undertaken the journey across the Greenland ice.

Note, September 22nd.-Baron Nordenskjöld has just returned, and, as might have been expected, he found the interior of Greenland a desert of ice, with the icy plain gradually sloping upwards to the interior. The ice rose at the furthest spot reached ( 280 miles from the coast) to 7000 feet above the sea, and was still seen to rise to the east. The results of the expedition are, however, of the most important character, confirming, as they do, the true theory of continental ice.

L. The Method of Least Squares. By F. Y. EDGeworth, M.A., Lecturer on Logic at King's College, London*.

I.

7 YHE Law of Error and the Method of Least Squares do 1 not traverse the same ground; and the direction of the one course of reasoning is inverse to that of the other. In the former we derive the formation of a source $\dagger$ of error from the confluence of an indefinite number of small tributaries. In the latter we start from a position lower down on the stream of causation, from observations resulting from a source of error, and reason up from given observations (accompanied with some knowledge of the source of error from which they result) to (a more complete knowledge of) the source of error, the facility-curve under which the observations range themselves. For example:-Given $x_{1}, x_{2}$, \&c. observations diverging according to a probability-curve of known modulus, but unknown centre, to determine the centre. These distinctions are likely to recommend themselves to those who have studied Mr. Glaisher's article and other first-rate anthorities. But there is another distinction, more interesting to the philo-

* Communicated by the Author.

$\dagger$ According to the received view, one particular source-the probabilitycurve; according to our views, a great variety of facility-curves (see previous paper). 
sopher and less familiar to the mathematician, namely, that in the Law of Errors we are concerned only with the objective quantities about which mathematical reasoning is ordinarily exercised; whereas in the Method of Least Squares, as in the moral sciences, we are concerned with a psychical quantity - the greatest possible quantity of advantrge. To illustrate this application of mathematics to psychical quantity is the primary object of the following paper; a secondary purpose is to classify the problems falling under our title (taken in a wide sense), and to offer some contributions towards their solution.

In order to attain the first object it is not necessary to go much beyond Laplace's Method of Least Squares. In the problem of Book II. art. 20 Laplace in effect, if not very explicitly, assumes that the sought result may be regarded as a linear function of the observations. He posits this form of the result, not assuming that the most probable value expressed in terms of the observations will be a linear function of the observations, which is in fact not generally true, but selecting the linear form as most advantageous, advantageous in respect of convenience to the calculator and avoidance of trouble. The linear form being assumed, Laplace goes on to determine the values of the constants. He decides in favour of the system of values which are inversely proportional to the respective mean squares of error upon two grounds, of which the second is bere regarded as the more fundamental-namely, that system of values is to be preferred which minimizes the disadvantage incurred in the long run by employing any particular system of values. Laplace takes as the measure of this integrated disadvantage the mean error. Gauss (dissenting from Laplace on what may seem almost trivial grounds) takes as the "moment" of error the measure of detriment incurred in the long run, the mean square. And it is conceivable that another criterion, which, in comparison with that of Laplace and Gauss, may be described as the mean zero power (corresponding to that system which, as compared with other linear systems, affords the most probable value), may have heen assumed by some, not as a first principle (Mr. Glaisher's view, to be presently considered), but as a derivative principle, as the measure of disadvantage.

It is here submitted that these three criteria are equally right and equally wrong. The probable error, the mean error, the mean square of error, are forms divined to resemble in an essential feature the real object of which they are the imperfect symbols-the quantity of evil, the diminution of pleasure, incurred by error. The proper symbol, it is submitted, for 
the quantity of evil incurred by a simple error is not any power of the error, nor any definite function at all, but an almost arbitrary function, restricted only by the conditions that it should vanish when the independent variable, the error; vanishes, and continually increase with the increase of the error. The proper symbol for the disadvantage incurred in the long run is an integral whose elements involve as factors the said arbitrary function. Advancing, then, in the direction indicated by Laplace and Gauss, let us designate the disadvantage of a single error by the symbol $\mathrm{F}\left(x^{2}\right)$, where $x$ is the amount of error and $\mathrm{F}$ continually increases with $x$. Or, if the detriment is not a symmetrical function of error, is not equal for the same extent of error, whether it be in excess or defect, put $\mathrm{F}(x)$ for the right-hand value of $x$, and $f(x)$ for the left-hand value of $x ; x$ being taken as positive in both cases. Now suppose we have adopted some particular system of values for the constants $\gamma_{1}, \gamma_{2}$, \&c. Then, by the law of errors, if we make several sets of observations, say

the quantities

$$
\begin{array}{cccc}
x_{1} & x_{2} & x_{3} & \& \mathrm{c} . \\
x_{1}^{\prime} & x_{2}^{\prime} & x_{3}^{\prime} & \& \mathrm{c} . \\
x_{1}^{\prime \prime} & x_{2}^{\prime \prime} & x_{3}^{\prime \prime} & \& \mathrm{c} ., \\
& \& \mathrm{c} . & & \& \mathrm{c} .
\end{array}
$$

$$
\begin{gathered}
\gamma_{1} x_{1}+\gamma_{2} x_{2}+\gamma_{3} x_{3}+\& c . \div S \gamma \\
\gamma_{1} x_{1}^{\prime}+\gamma_{2} x_{2}^{\prime}+\gamma_{3} x_{3}{ }^{\prime}+\& c . \div S \gamma, \\
\text { \&c. } \div \text { \&c. }
\end{gathered}
$$

will be ranged under a probability-curve of the form $\frac{1}{\sqrt{\pi c}} \epsilon^{-\frac{x^{2}}{a^{2}}}$, where $\alpha$ is a known function of the sought quantities $\gamma_{1}, \gamma_{2}$, \&c. We have now to take $\alpha$ so that the total disadvantage in the long run of an indefinite number of sets of observations may be a minimum. This total disadvantage is

$$
\int_{0}^{\infty} \frac{1}{\sqrt{\pi} \alpha} \epsilon^{-\frac{x^{2}}{a^{2}}}[\mathrm{~F}(x)+f(x)] d x
$$

Put $x=\alpha \xi$. The quantity which it is proposed to minimize becomes

$$
\int_{0}^{\infty} \frac{1}{\sqrt{ } \pi} \epsilon^{-\xi^{2}}[\mathrm{~F}(\alpha \xi)+f(\alpha \xi)] d \xi
$$

a being regarded as variable, the first term of variation

* Glaisher, op. cit. 
becomes

$$
d \alpha \int_{0}^{\infty} \frac{1}{\sqrt{\pi}} \epsilon^{-\xi^{2}}\left[\mathrm{~F}^{\prime}(\alpha \xi)+f^{\prime}(\alpha \xi)\right] \xi d \xi
$$

Every element of this integral is positive. Therefore the integral is positive. Therefore the propositum, the disadvantage, continually increases as $\alpha$ increases. Therefore it is the least possible when $\alpha$ is the least possible. Which was to be demonstrated.

By an extension of the preceding reasoning we obtain the following fundamental theorem. One instrument (or one method of using the same instrument, one method of treating given observations) is to be preferred to another, when, $\phi_{1}(x)$, $\phi_{2}(x)$ being the facility-functions expressing the divergence in the first and second cases respectively from the real point, $\int_{0}^{x} \phi_{1}(x) d x$ is greater than $\int_{0}^{x} \phi_{2}(x) d x$ for every value of $x$.

It may be objected that these results might better have been grounded on the more solid and objective foundation of greatest probability rather than greatest advantage; agreeably to Laplace's first view as formulated by Mr. Glaisher*namely, it being assumed that the quantity to be measured is accurately determined, if its error lies between zero and infinitesimal $k$, that system of factors which renders the probability that the result obtained by means of them is accurately determined greater than the probability of a result obtained by means of any other system of factors is to be preferred. It may be replied that the principles of greatest advantage and greatest probability do not coincide in general; that here, as in other departments of action, when there is a discrepancy between the principle of utility and any other rule, the former should have precedence.

'To exhibit this discrepancy it suffices to observe that the disadvantage which it is proposed to minimize is the loss of utility, the quantity of pain due to an erroneous measure being employed in practice, in the arts. Why is this evil a minimum when the probability of our measurement being within the distance $k$ of the real quantity is a maximum? Let us take a simple, although grotesque, example. Here are two shoemakers competing for the contract to supply an army with boots. Other things being equal, we have to select him who makes the best fits, who minimizes the disadvantage expressed by Horace:-

$$
\text { ". ... ut calceus olim, }
$$

Si pede major erit, subvertet ; si minor, uret."

* Memoirs of the Royal Astronomical Society, xl. p. 101. 
Is the shoemaker who makes a rather greater proportion of exact fits (who has a smaller probable error), but when be does make a misfit makes a terribly painful one, necessarily the most advantageous? To quote Horace* again, the slave who, in spite of his many accomplishments, "semel latuit" ("once and but once I caught him in a lie," as Pope turns it), may have had, so to speak, a less "probable error," and yet may have been less "advantageous" to his master than one who had a greater number of less serious faults. The man who seldom tells a lie, but when he does "lies like a man," may do more harm than the habitual dealer in white lies. I am aware that there is something paradoxical in the preceding illustrations ; but I submit that it would be affectation in a mathematical writer not occasionally to glance at the real objects to which his ideal conceptions are applied, and that the disadvantages just instanced are quite homogeneons with, only more familiar than, the disadvantage due to the employment in the arts of erroneous measurement (e. g. the disadvantage of astronomical mismeasurement), the disadvantage which, according to Laplace and Gauss, it is the object of the calculus to minimize.

The following is a more dignified example. Here are two instruments of observation, the errors incurred by which are ranged respectively under a probability-curve with modulus unity, and under the facility-curve $y=\frac{1}{\pi} \frac{c}{c^{2}+x^{2}}$, where $c$ is small. Which of these instruments is to be preferred? According to Mr. Glaisher's test, unquestionably the latter. But, according to our view, it may well happen that the disadvantage in the long run is in the former case finite, in the latter case infinite: for example, if the disadvantage dependent upon a particular error may be expressed as any power (not less than the first), or sum of powers, of the extent of error.

Nor, again, does the principle of greatest probability as compared with the principle of greatest utility give a consonant answer in the following case:-Given the law of error of a certain instrument, is it better to make a practice of confining ourselves to a single observation, or of proceeding to an average? Upon Mr. Glaisher's view, if the average facility-curve is such as to have its maximum value (its head, so to speak) above the primary curve, the average must be preferable to the simple observation. This also follows from our first principle (by the theory of p. 363) when there is only one intersection between the primary and average semi-curves; but not quite generally. Suppose that there are three inter-

* Ep. II. 2. 
sections on each side of the origin, and that the space on each side of the origin is divided by ordinates (one at the origin, one at infinity, and two intermediate) into three compartments such that for each compartment the area bounded by the abscissa, ordinates, and primary curve is equal to the area bounded by the same right lines and the average curve. Suppose, further, that throughout the first and third compartments the disutility-function is almost level, the disadvantage of a particular error only just increases with the extent of error, while in the second compartment the same function rises steeply. Then the appropriate mathematico-psychical reasoning will show that it is better to abide by a single observation than to take an average.

The two preceding examples, to which it would be easy to add others similar, are not put forward as practically inportant, but rather (like the imaginary cases put by Hume in his inquiry concerning moral sentiments) as assisting us to distinguish the general principle from the particular rule, and in the act of doing so to discern the supremacy of the principle of utility.

We have next to review some typical instances of the problems solved by the method of least squares. Let us take as the principle of a rough classification, complexity. As a first division we may demarcate those cases in which there is but one measurable; a single quantity $x$, whereof $x_{1}, x_{2}$, \&c. are values, or more generally functions, given. 'This class may be subdivided according as the facility-curves which generate $x_{1}, x_{2}$, \&c. are (I.) or are not (II.) symmetrical. In the simpler cases we need not take the trouble of distinguishing between the most probable and most advantageous values, since they are coincident. But the distinction soon emerges.

Subclass I. may be subdivided according as the symmetrical facility-curves under which the observations are ranged are (A) or are not (B) Probalility-curves.

I. A (1) The following is one of the simplest problems which our subject presents:-Given a set of observations $x_{1}$, $x_{2}$, \&c., and given that they have been generated by divergence from an unknown point according to one given law of error, a probability-curve of given modulus, to find the most probable (and advantageous) value for the unknown point. By a familiar application of the differential calculus the sought value is the mean of the observations.

A variant of this problem is when the observations are distributed into groups, each of which diverges from one and the same unknown point according to different given probabilitycurves. The solution is of course the weighted mean. 
Another variant in this and, be it said once for all, all the subsequent typical problems, is when the observations stand for, not the simple value of, but definite functions of, the real quantity.

I. A (2) Given a set of observations $x_{1}, x_{2}$, \&c., and given that they were generated by divergence according to a given curve of probability of which the modulus is not given but sought. Let $c$ be sought modulus; then it must be taken so that $\left(\frac{1}{c}\right)^{n} e^{-\frac{x_{1}^{2}+x_{2}^{2}+\& c .}{c^{2}}}$ should be a maximum; whence

$$
\frac{c^{2}}{2}=\frac{\mathrm{S} x_{r}^{2} *}{n} \text {. }
$$

A variant would be the case when the observations are distributed into groups, each with a different modulus, and the ratio between the moduli is given.

I. A (3) By the degradation of the data in either of the preceding problems we reach the complex problem :Given a set of ohservations $x_{1}, x_{2}, \& c$., and given that they have been generated by divergence according to one and the same probability-curve from a single point, but given neither that point nor the modulus, to find both. Put as the probability of the concurrence of a particular central point and a particular modulus

where

$$
\frac{\mathrm{P} d \xi d c}{\int_{0}^{\infty} \int_{-\infty}^{\infty} \mathrm{P} d \xi d c}
$$

$$
\pi^{\frac{2}{n}} \times \mathrm{P}=\left(\frac{1}{c}\right)^{n} \times \epsilon^{-\left[\frac{\left(x_{1}-\xi\right)^{2}+\left(x_{2}-\xi^{2}+\& c_{c}\right.}{c^{2}}\right]} .
$$

Equating to zero the first term of variation (that is, equating to zero the coefficient of $d \xi$ and also the coefficient of $d c$ in the development of the above propositum), we have two equations to determine $\xi$ and $c$. Whence the most probable value of $\xi$ is the mean of $x_{1}, x_{2}, \& c$, and the most probable value of $c$ is the square root of twice the mean square of apparent errors, viz.

$$
\sqrt{2\left[\frac{\left(\xi-x_{1}\right)^{2}+\left(\xi-x_{2}\right)^{2}+\& \mathrm{c} .}{n}\right]} ;
$$

a solution of the compound problem which follows the analogy of the simple problems (1) and (2), and which holds whether the number of observations be finite or infinite.

I believe that this conclusion is usually restricted to the

* Cf. Merriman, 'On Least Squares,' p. 143. 
case of an infinite number of errors ; that, for the finite case, there would usually be substituted $(n-1)$ for $n$ in the above value of $c$. It is with the greatest diffidence that I submit a statement which seems to be contradicted by the most distinguished authorities, including Gauss and Airy*; and only not contradicted, but not, as far as I remember, asserted, by Laplace and Poisson. Perhaps I have misunderstood the quæsitum proposed by Airy, Merrimant, and other writers. For I assert with some confidence that (in spite of the rather puzzling expressions of some authors) the procedure above employed to determine the maximum of an expression involving two independent variables is correct. I submit also that the only significant, or at least the most important, quæsita afforded by the case in hand are either what was enounced above, or what is the "probable error" $\ddagger$ incurred by taking the mean of the observations as the real value. To investigate this probable error, it is proper to consider the given observations as generated by one of an indefinite number of constitutions corresponding to the different values of $\xi$ and $e$ 's, each of which operates in the long run a number of times proportionate to $\frac{\mathrm{P} d c d \xi}{\mathrm{SP}}$; that is, if we take the origin at the mean point of the observations, and put $c=\frac{1}{h}$ proportionate to

$$
\frac{\frac{h^{n}}{\pi^{n}} \epsilon^{-\left[n \xi^{2}+\mathrm{S} x^{2} r\right]^{2}} d h d \xi}{\int_{-\infty}^{\infty} \int_{0}^{\infty} \mathrm{P} d h d \xi} .
$$

It may be observed that when $h$ is constant this expression reduces to

$$
\frac{1}{\sqrt{\pi}} \sqrt{n h} \epsilon^{-n h^{2} \xi^{2}}
$$

from which we see that in case (1) the probable error incurred by taking the average of operations is the unit probable error, as it may be called, 476 multiplied by $\frac{h}{\sqrt{n}}$; as was to be oxpected. But in case (3) this analogy is deserted. It appears from the preceding expression, that the number of times we

* $\$ \$ 58-60$.

† 'Least Squares.'

f Cf. Merriman, 'Least Squares, pp. 27 \& 146 
shall make an error of $\xi$ in taking the mean as the true value is

of the form

$$
\frac{\int_{0}^{\infty} \mathrm{P} d h}{\int_{-\infty}^{\infty} \int_{0}^{\infty} \mathrm{P} d h d \xi} \cdot d \xi
$$

$$
\frac{\mathrm{J}}{\left(\mathrm{S} a_{r}^{2}+n \xi^{2}\right)^{\frac{n+1}{2}}} \text { (say } n \text { even). }
$$

The errors committed by taking the mean as the real value are ranged under the above* sub-exponential expression. Whence it appears that what may be called the measure of the probable error is the sum of the squares of the apparent errors divided by $n$, not, as some might seem to imply, by $(n-1)$; although this modulus, as I think it may be called with propriety, is not to be multiplied by the usual factor 476 , but by the length of the abscissa which halves the half-area of the curve just indicated.

What is frequently said in favour of the expression $2 \frac{\mathrm{S}\left(\xi-x_{r}\right)^{2}}{n-1}$ (where $\xi$ is the mean of the observations) instead of $2 \frac{S\left(\xi-x_{r}\right)^{2}}{n}$ in the present and similar problems, namely, that the latter expression, the sum of squares of apparent errors, is certainly less than the sum of squares of real errors, appears to be true but not pertinent. For what have we to do with the sum of squares of real errors, except as a mark of one or other of the quæsita above proposed?-(1) The law according to which the observations diverged, the modulus of the probability-curve, from the groups constituted by which the observations are regarded as random selections; (2) the errors incurred by taking the mean as the real value It may be observed, too, that the most probable value (as deduced from the observations) of the sum of squares of real errors is the sum of squares of apparent errors ; a statement which is quite consistent with the admission that this latter value is certainly (infinitely probably) less than the real value. The case might be compared to the following :-A random selection of an abscissa being made from the group indicated by the curve $y=\frac{1}{\sqrt{\pi} k} \epsilon^{-\frac{x^{2}}{k^{2}}}$, required the most probable value

* See Phil. Mag. Oct. 1883, p. 306. 
of the square of the selected abscissa. It is zero. Yet it is infinitely probable that the real value is greater than zero.

It appears from the subexponential form* incidental to this problem, that if, after taking the mean of a group of observations of the type I. A. 3, we take the mean of a second group of the same type, the mean of those two means (or of any number of such means) is not likely to be as much nearer the true value as might have been expected.

A variant of Prob. (3) is obtained by the distribution of the observations into groups each of the type (3). This variant might be obtained from the variant of Prob. I. Thus, supposing the limits of the groups still given, let our knowledge of the weight corresponding to each group be degraded through all degrees of conjectural knowledge to absolute uncertainty. Now let the barriers which separate the groups be unfixed, and finally become absolutely uncertain, and we shall have reached a fourth case,

I. A 4, in which we do not know the weight of any of the observations; and they may all, for all we know, have different weights. The solution may be reserved till we reach the more general case of I. B. 4 .

I. B. Facility-curves which are symmetrical†, but not probability-curves, present similar cases for the exact solution of which it would be necessary to assume as given some particular form, $e \cdot g \cdot \frac{1}{\sqrt{\pi c}}\left(\alpha+\frac{2 \beta}{c^{2}}\right) \epsilon^{-\frac{x^{2}}{c^{2}}}$, where $\alpha+\beta=1$; unlessindeed, as Donkin has observed $\ddagger$, it were possible by an unimaginable perfection of the calculus of functions to take, as it were, a mean of all admissible functions.

Approximate solutions of cases under this heading are given by the Method of Least Squares, as discovered by Laplace. Concerning these it may be observed that, if the subexponential form is an appreciable ingredient of the elemental facilitycurves, then the factors assigned by the method of least squares are not in general the most advantageous. It is obvious to object that the number of the observations in the case to which the Method of Least Squares is applicable is supposed infinite; and therefore that the principle mentioned in the Postscript of the previous paper will apply. But then infinite is to be interpreted here as very large. Or, rather, not so very large; as we are told in recommendation of this very theorem whose

* See previous paper.

+ Some of the following inquiries can only by courtesy be included under the term Least Squares.

† Phil, Mag. [4] vol. ii, p. 56:

Phil. Mag. S. 5. Vol. 16. No. 101. Nov. 1883. 
accuracy I impugn. How, then, can we be certain that the effect of a subexponential ingredient is extinguished?

Agreeably to the distinction clearly exhibited by Mr. Glaisher (op. cit. p. 102 foot, and p. 103), the method of least squares belongs to $B(3)$ or $B(4)$ according as it is, or is not, given that all the observations have the same weight. The latter case presents the much vexed question, What shall be done with considerable outlying errors? Shall the exceptional observation be omitted from the average, as Peirce* says? Or shall it count for one, as Airy sayst? Or shall it count indeed, but not count for one, as says DeMorgan? Upon the hypothesis here entertained DeMorgan's view is undoubtedly correct in theory, though in practice it may not differ from the practice of Peirce. The approximative method is justified in following the analogy of the exact method; which would, according to the inverse method here all along contemplated, include all the data among the premises, though it may be that the conclusion, which is a function of them all, is less affected by (the variation or omission of) some than others. Thus, to take an example transferred here from its proper place (A 4): suppose we have two observations, $x_{1}$ and $x_{2}$, close together, and a third outlying, given that the generating facility-curves are probability-curves, but nothing further. Then we have to determine $x, h_{1}, h_{2}, h_{3}$, so that

$$
\pi^{-\frac{3}{2}} h_{1} h_{2} h_{3} \epsilon^{-\left[h_{1}^{2}\left(x-x_{1}\right)^{2}+h_{2}{ }^{2}\left(x-x_{2}\right)^{2}+h_{3}{ }^{2}\left(x-x_{3}\right)^{2}\right]}
$$

is a maximum. Whence

(1) $1-2 h_{1}^{2}\left(x-x_{1}\right)^{2}=0$,

(2) $1-2 h_{2}^{2}\left(x-x_{2}\right)^{2}=0$,

(3) $1-2 h_{3}^{2}\left(x-x_{3}\right)^{2}=0$,

(4) $h_{1}^{2}\left(x-x_{1}\right)+h_{2}^{2}\left(x-x_{2}\right)+h_{3}^{2}\left(x-x_{3}\right)=0$.

Whence

$$
\frac{1}{\left(x-x_{1}\right)}+\frac{1}{\left(x-x_{2}\right)}+\frac{1}{\left(x-x_{3}\right)}=0 \text {. }
$$

Of the roots, that one is to be selected which makes a maximum

or, as

$$
h_{1} h_{2} h_{3} \times e^{-h_{1}{ }^{2}\left(x-x_{1}\right)^{2}-h_{2}{ }^{2}\left(x-x_{2}\right)-h_{3}{ }^{2}\left(x-x_{3}\right)^{2}} ;
$$

$$
l_{1}^{2}=\frac{1}{2\left(x-x_{1}\right)^{2}}
$$

* Astron. Journ. vol. ii. p. 161 (Cambridge, America).

$\dagger$ 'Astronomical Journal,' vol. iv. p. 137. 
that which makes a maximum,

$$
\frac{1}{\left(x-x_{1}\right)\left(x-x_{2}\right)\left(x-x_{3}\right)} \text {. }
$$

When two observations are close together, the root of $x$ which lies close to the two observations is evidently the appropriate value. The weight of those two observations is then high; the weight of the third observation, if it lies at a distance from the other two, is small. These considerations may easily be extended to the case of any number of observations given by probability-curves. The equation

$$
\frac{1}{x-x_{1}}+\frac{1}{x-x_{2}}+\text { \&c. }+\frac{1}{x-x_{n}}
$$

is easily formed, and admits of approximative treatment for one or two observations remote from the majority ; and doubtless in other cases.

In case of laws of error other than probability-curves, the method of course does not afford the most probable value. But I submit that the method is still a very advantageous method; more advantageous both in respect of accuracy and convenience than the method proposed by DeMorgan and $\mathrm{Mr}$. Glaisher. Our method is at least accurate in one case, and that the very important and typical case of the law of error being a probability-curve; comparable in that respect to $\mathrm{La}_{2}$ place's treatment of the sought value as a linear function of the observations; a procedure which leads to the most probable value in the case of probability-curves, but is in general only a convenient, an advantageous, procedure*. But their method is never (except by chance) accurate, and always inconvenient. As I understand Mr. Glaisher, if three observations of unknown weight are given; say $x_{1}, x_{2}, x_{3}$, his procedure is first to take the mean $\frac{x_{1}+x_{2}+x_{3}}{3}$; then to form the apparent errors $\frac{x_{2}+x_{3}-2 x_{1}}{3} \& c . ;$ then to form what may be called the provisional weight (for all the observations),

Then put

$$
h^{2}=\frac{1}{2\left[\left(\frac{x_{2}+x_{3}-2 x_{1}}{3}\right)^{2}+\& c .\right]} \text {. }
$$

$$
p_{1}=\frac{1}{\sqrt{\pi} \sqrt{\overline{2}}\left[\frac{\left(x_{2}+x_{3}-2 x_{1}\right)^{2}}{3}+\& c .\right]} \epsilon^{-h^{2}\left(\frac{x_{2}+x_{3}-2 x_{1}}{3}\right)^{2}} .
$$

$2 \mathrm{D}_{2}$ 
Let $p_{1}^{2}$ be the new provisional weight of the first observation. Then make

$$
p_{1}^{2}\left(x-x_{1}\right)^{2}+p_{2}^{2}\left(x-x_{2}\right)^{2}+p_{3}^{2}\left(x-x_{3}\right)^{2}
$$

a minimum. Let $x$ be the new (weighted) mean. Calculate a new $p_{1} \& c$. , and continue the process until you come to a standstill.

It may be questioned whether this is exactly the procedure which DeMorgan had in view. Would not his language apply to the following process? Find, as before, the prim $\hat{a}$ facie value $\frac{x_{1}+x_{2}+x_{3}}{3}$; then calculate the weights, upon the principles herein adopted, on the hypothesis that the putative is the real point; and that the apparent errors are the result of divergence therefrom, according to probability-curves of different moduli. The weights* are

$$
\begin{aligned}
h_{1}^{2} & =\frac{1}{2\left(\frac{x_{2}+x_{3}-2 x_{1}}{3}\right)^{2}} \\
\& \mathrm{c} . & =\quad \& \mathrm{c} .
\end{aligned}
$$

Then make $h_{\mathrm{I}}^{2}\left(x-x_{1}\right)^{2}+h_{\mathrm{z}}^{2}\left(x-x_{2}\right)^{2}+h_{\mathrm{g}}^{2}\left(x-x_{3}\right)^{2}$ a minimum,

$$
x=\frac{\frac{x_{1}}{\left(x_{2}+x_{3}-2 x_{1}\right)^{2}}+\frac{x_{2}}{\left(x_{3}+x_{1}-2 x_{2}\right)^{2}}+\& c .}{\frac{1}{\left(x_{2}+x_{3}-2 x_{2}\right)^{2}}+\quad \text { \&c. }}
$$

Find a new set of weights, and so on.

Neither process leads directly, in the case of probabilitycurves, to the most probable value, which is

$$
\text { The Mean } \pm \sqrt{\left(x_{1}^{2}+x_{2}^{2}+x_{3}^{2}-\left(x_{1} x_{2}+x_{2} x_{3}+x_{3} x_{1}\right)\right.} \text {. }
$$

Query whether the DeMorgan-Glaisher process would ultimately reach the most probable value. Their method, then, appears to be less advantageous in respect of accuracy than the method here suggested. Their method would perhaps be more advantageous in respect of convenience in some cases where the real weights are nearly equal, and not many approximative steps are required. It must be remembered, however, that in these cases, as our analysis shows, the observations must be close together; and therefore that our method also becomes facilitated in this case. For instance, taking the mean of the observations as origin, neglect powers higher than the second of $x_{1}, x_{2}$, \&c. measured from this origin.

* See I. A. (2). 
Then the general equation for $x$ becomes

$$
n x^{n-1}-(n-1) \Sigma x_{r} . x^{n-2}+(n-2) \sum x_{r} x_{s} x^{n-3}
$$

which reduces to a quadratic. And in the general case the coefficients of the equation of the $(n-1)$ th degree, consisting of combinations of the observations, might possibly be evaluated by a calculating machine. Whereas the alternative methods do not seem equally to lend themselves to mechanical treatment. It may be questioned, therefore, whether even in respect of convenience the DeMorgan-Glaisher method has the advantage.

II. The difficulties thicken as the hypotheses become more rarefied. For unsymmetrical facility-curves the most probable value is not in general, even presumably, coincident with the most advantageous value. The most probable value may, of course, be determined if we assume a particular form of facility-curve, and determine by inverse calculations analogous to those already given the value of the constants. It should be observed that we must here expressly assume, what before was taken for granted, that is, not only what the form of each generating facility-curve is, but also how it is disposed with regard to the real point. Whereas in unsymmetrical curves the longest ordinate, the centre of gravity, the bisection of the area, may all correspond to different abscissæ, which of them, if any of them, corresponds to the real point?

The scruple just raised affects also that approximative method of determining the most advantageous value, which is afforded by Poisson's extension of what may be called Laplace's law of errors; I mean Poisson's proof that, even in the case of unsymmetrical curves, the several values of a linear function of observation, $\frac{\gamma_{1} x_{1}+\gamma_{2} x_{2}+\& c .}{\gamma_{1}+\gamma_{2}+\& c_{.}}\left(x_{1}, x_{2}\right.$, \&c. being numerous), may be regarded as ranged under a probability-curve, whose centre is the mean of the mean errors of the elemental curves. If now each facility-curve be so disposed about the "real point" (as, waving metaphysical difficulties, I have called it), then indeed the weighted mean* will be the most advantageous value; but not in general. Poisson himself, as I understand him, points out this cause of failure (Connaissance des Temps, 1832 "Suite," $\$ 9$ beginning).

A fresh difficulty, connected with a fresh quæsitum, now also makes its appearance. We have all along described the method of least squares (taken in a large sense) as an inverse

* Assuming of course that the utility-functions are symmetrical, and that the influence of subexponential elements may be neglected. 
process by which we remount from measurements to the measurable, from the plural manifestations of a thing to the thing itself. But now there becomes differentiated another quxesitum which has hitherto been coincident with the above; viz. given $n$ manifestations (observations), what is the (most probable or most advantageous) value of the $(n+1)$ th manifestation? For example, if the positions of $n$ shot-marks on a target are reported to me, and it is given that they are all results of firing at a wafer, I can in my study calculate (1) the most probable position at which the shots were fired; (2) (supposing that the firing is renewed after the $n$ observations have been noted) the point most likely to be hit by the $(n+1)$ th shot. When the law according to which the shots diverge right or left of the bulls-eye is symmetrical, these quæsita are identical, but not otherwise.

Continually degrading our data concerning the genesis of the observations supplied to us, as hypothesis after hypothesis unwinds, we should come to the very zero of assumptionabsolute ignorance as to the genesis of $x_{1}, x_{2}$, \&c. In this case many of the preceding problems become unmeaning. But not the least important-what is the most probable (or advantageous) value of the $(n+1)$ th observation-may still be asked. An answer, which is probably not altogether valueless, but serviceable as a starting-point for hypotheses, is afforded by the remarkable method explained by Boole in the 'Proceedings of the Royal Society of Edinburgh;' especially if we admit, what Boole does not admit, that the value of a quite unknown constant expressing probability is to be treated as $\frac{1}{2}$; as Donkin* contends. Both Donkin's assumption and those upon which the whole of Boole's new method of probability is grounded are not to be regarded as arbitrary, but as the solid result of experience-that constants do in general in rerum natur $\hat{a}$ as often present one value as another. This principle is illustrated by the occurrence of one digit as often as another in natural constants (a fact actually verified by Mr. Proctor in the case of logarithms). The principle is verified and shown to be at least a good working hypothesis by the fact that it underlies all the methods of least squares. For they all presuppose that the a priori probability of the real quantity, the quæsitum, being, say, between $a$ and $a+\Delta a$, is the same as the $\dot{a}$ priori probability of its being between $b$ and $b+\Delta b$-between formally infinite and practically considerable limits. And this supposition of equal à priori probability can have no significance, as $\mathrm{Mr}$. Vennt well

* Phil. Mag. [4] vol. i.

+ 'Logic of Chance,' chap. vi. 
reasons, unless it mean that measurables do, as a matter of fact, as often have one value as another. But, in touching these considerations, we have already passed the frontier whick separates mathematical reasoning from general philosophy.

It remains only to add that the preceding problems are immensely complicated when, instead of our single variable it, there are several variables; but that these additional difficulties have been triumphed over by a long line of distinguished mathematicians, from Laplace to Glaisher.

LI. Description of an Apparatus to illustrate the Production of Work by Diffusion. By C. J. Woodward, B.Sc.*

$\mathrm{D}^{1}$ IFFUSION, as a source of energy, is usually shown in the lecture-room by bringing a jar of hydrogen over a porous vessel fitted up with a glass tube dipping into water. Hydrogen, by inward diffusion, enters the jar; the internal pressure thus produced forces the water down, and a stream of bubbles escapes from the tube. On removing now the jar of hydrogen, outward diffusion of the hydrogen takes place, a minus pressure is produced in the porous vessel, and the water is lifted.

The apparatus I am about to describe is an adaptation of this experiment to the production of an oscillatory movement of a beam from alternate inward and outward diffusion of hydrogen.

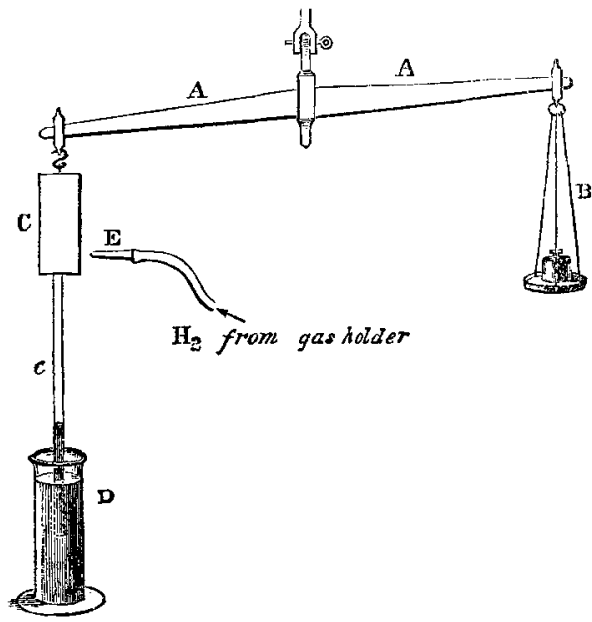

The apparatus is represented in the annexed figure. A A is a scale-beam about 3 feet long, carrying at one end a scale-

* Communicated by the Physical Society, having been read at the Meeting on May 12th, 1883. 